\title{
Maintaining immunological tolerance with Foxp3
}

\author{
Lauren E Mays ${ }^{1}$, Youhai H Chen ${ }^{1}$ \\ ${ }^{1}$ Department of Pathology and Laboratory Medicine, School of Medicine, University of Pennsylvania, Philadelphia, PA 19104, \\ USA
}

Central tolerance in the thymus is the primary mechanism for deleting autoreactive T cells. Despite this, escape of self-reactive T lymphocytes into the periphery reveals the threat of autoimmunity. To compensate for its imperfection, the thymus also produces a naturally occurring subset of Foxp3 + CD4+CD25+ regulatory $\mathrm{T}$ cells with suppressive function, capable of controlling autoreactive cells. Foxp3 (forkhead box P3), the lineage-specific marker for this subset of cells, is crucial to their thymic development and peripheral function, and yet the transcriptional program driven by Foxp3 was until now largely undefined. Emerging evidence has provided insight into its role: from the ability of Foxp3 to cooperate with other transcription factors such as NFAT, to the genome-wide characterization of target genes directly bound and regulated by Foxp3. Here we discuss the discovery of naturally occurring regulatory T cells - their phenotype, development, maintenance, and function - largely as they are defined by the lineage-specific marker, Foxp3.

Keywords: Foxp3, tolerance, T cell, suppression, regulatory cells

Cell Research (2007) 17:904-918. doi: 10.1038/cr.2007.84; published online 19 October 2007

\section{T-cell receptor (TCR) rearrangement, tolerance, and autoimmunity}

The primary role of the immune response is to protect the body from foreign threats. This requires the immune system to recognize a countless number of pathogens and retain the ability to differentiate between 'self' and 'non-self' [1]. The breadth of this response is attributed to the genetic diversity of BCRs and TCRs which provides an extensive repertoire of immune effector cells. In the case of T lymphocytes, immature T cells in the thymus contain multiple TCR $\alpha$ and $\beta$ chain genes, each composed of multiple gene segments. $\mathrm{V}(\mathrm{D}) \mathrm{J}$ recombination determines which TCR $\alpha$ and $\beta$ genes are combined, during which the respective segments of

\footnotetext{
Correspondence: Lauren E Mays ${ }^{1}$, Youhai H Chen ${ }^{2}$

${ }^{1}$ Department of Pathology and Laboratory Medicine, University of Pennsylvania School of Medicine, 125 S. 31st Street, Philadelphia, PA 19104, USA.

Tel: +1 215898 8359; Fax: +1 2158986588

E-mail: maysle@mail.med.upenn.edu

${ }^{2}$ Department of Pathology and Laboratory Medicine, University of Pennsylvania School of Medicine, 713 Stellar-Chance Labs, 422 Curie Blvd., Philadelphia, PA 19104, USA.

Tel: +1 215898 4671; Fax: +1 2155733434

E-mail: yhc@mail.med.upenn.edu
}

each gene are pieced together by the RAG recombinase to provide an intact TCR [2]. The critical role of the thymus, however, is not in generating an extensive repertoire of TCRs, but in deleting those that are autoreactive. Thus, the specificity of the immune response for 'non-self' depends on central tolerance, whereby over $95 \%$ of the immature $T$ cells generated in the thymus undergo negative selection to rid the body of autoreactive cells [3].

Once the pre-T cell is expressing an intact receptor, it scans the thymus for cognate antigen presented in selfMHC molecules. The TCR is restricted to interact with MHC I or MHC II, thus dedicating the T cell to become either CD8+ or CD4+, respectively [4]. With help from the thymic transcription factor AIRE (autoimmune regulator), thymic epithelial cells ectopically express low levels of selfantigens from organs throughout the body [5]. Most, but not all, peripheral self-antigens are present in the thymus. If the TCR of an immature T cell binds self-antigen and self-MHC with low affinity, it receives a signal for maturation and undergoes 'positive selection' [6]. These T cells will go on to recognize foreign antigens in self-MHC when they reach the periphery as mature $\mathrm{CD} 4+$ and $\mathrm{CD} 8+\mathrm{T}$ cells. Immature $\mathrm{T}$ cells that recognize self-antigens in self-MHC with high affinity receive a stimulus that is too overwhelm- 
ing for the cell, inducing death. This is the process of clonal deletion or 'negative selection' of autoreactive cells. If the TCR interaction is somewhere in between the endpoints of positive and negative selection, the $\mathrm{T}$ cell reacts with the self-antigen but escapes negative selection. Instead of being deleted, these cells display an anergic phenotype in the periphery, exhibiting decreased proliferation and cytokine production following TCR engagement. Finally, because not all self-antigen genes are activated by AIRE in the thymus, some immature autoreactive $\mathrm{T}$ cells never engage with self-antigens. These cells avoid deletion and anergy, migrating to peripheral lymphoid organs as naïve 'ignorant' cells [7]. In the periphery these T cells are autoreactive and have the potential to cause autoimmunity if they become activated or are not properly suppressed.

Autoimmunity is the failure of the immune system to maintain tolerance against 'self'. Autoreactive T cells in the periphery recognize self-antigens as foreign and begin to attack the bodies' own tissue. In multiple sclerosis (MS), for example, the body attacks oligodendrocytes composing the myelin sheath which insulates neuronal axons. This inflammatory demyelinating disease interrupts electrical signaling and nerve impulses throughout the brain and spinal cord [8]. Experimental autoimmune encephalomyelitis (EAE), an animal model for MS, also presents as a demyelinating autoimmune disease [7]. In susceptible animals, EAE is induced following injection of a myelin antigen such as myelin basic protein (MBP), myelin oligodendrocyte glycoprotein (MOG), or proteolipid protein (PLP). EAE as a model of MS is one of many important tools essential to the study of the pathophysiology and immunology underlying autoimmune diseases.

\section{The role of self-antigen specific CD4+ $T$ cells in gen- erating autoimmunity}

In the late 1980s and early 1990s, investigators were addressing the causative factors of autoimmunity: whether the primary auto-reactive $\mathrm{T}$ cells were $\mathrm{CD} 8+$ or $\mathrm{CD} 4+$, whether pathogenesis required multiple factors working at once, and what controlled the activation of these cells. The idea that multiple types of $\mathrm{T}$ cells were simultaneously required for the development of autoimmunity was refuted by the finding that MBP-specific T-cell clones could transfer EAE to nude mice [9]. Studies in CD8-/- mice as well as those with anti-CD8 antibodies demonstrated that EAE develops at equal rates in the presence or absence of CD8 $[10,11]$. Thus, CD8+ T cells are not essential for the pathogenesis of EAE. In 1994, Lafaille et al. showed that EAE could occur in the absence of B cells and antibodies as well [12]. Therefore, autoimmune pathogenesis of EAE may be attributed to self-reactive CD4+ T cells alone. A series of observations made around this time support this theory: (1) self-antigen-specific CD4+ T cells and MHC class II presenting antigen-presenting cells (APCs) are present in inflamed tissues in MS patients [13]; (2) the disease is associated with certain MHC II alleles, suggesting that MHC II-restricted CD4+ T cells play a role in pathogenesis [14]; and (3) CD4 + T cells from healthy donors or patients can respond to self-Ag from the affected tissue in vitro [15]. Thus, in the absence of CD8+ T cells, CD4+ T cells do exhibit autoimmune potential.

Following antigenic stimulation, naïve $\mathrm{CD} 4+\mathrm{T}$ cells can differentiate into several different functional subtypes - Th1, Th2, Th17, and Treg - depending upon the microenvironmental cytokine milieu at the time of their differentiation, and the expression of lineage-specific transcription factors (Figure 1). Each subset is characterized by distinct cytokine production and effector function [16-18]. Before the discovery of the Th17 lineage, pro-inflammatory, IFN$\gamma$-producing, Th1-type CD4+ helper T cells were thought to play the major role in pathogenesis of autoimmune diseases such as multiple sclerosis or EAE, diabetes and rheumatoid arthritis. Mice that tolerate MBP and do not develop EAE demonstrate a decreased Thl response to MBP antigen [19]. On the other hand, EAE inducing MBP-specific T cells secrete Th1 type cytokines, and induce disease in naïve mice following adoptive transfer [20]. In addition, mice lacking Th1 transcription factors (T-bet and STAT-4) are resistant to the development of EAE [21, 22]. More recent evidence, however, suggests that Th17 cells are the primary contributors, acting as even more potent pro-inflammatory mediators $[23,24]$. Autoimmunity still occurs in IFN- $\gamma$ or IFN- $\gamma$-receptor deficient mice, which can be prevented by neutralization of IL-17 [25-27]. IL-17 is expressed in the target tissues of patients with numerous autoimmune diseases, and neutralization of this cytokine prevents the development of EAE $[28,29]$. It is not yet determined whether the roles of Th1 and Th17 cells in autoimmune pathogenesis are mutually exclusive. The observation that IL-17 expression is present during acute EAE, while IFN$\gamma$ increases and persists for a longer period in the CNS of these mice, suggests that perhaps both subsets cooperate to induce tissue-specific autoimmunity [30].

\section{Regulating autoimmunity: the presence of a sup- pressor cell}

As mentioned above, self-reactive CD4+ T cells can avoid central tolerance mechanisms in the thymus and escape to the periphery. In fact, these cells are present in the periphery of healthy animals, but rarely cause autoimmune diseases. Generally, they remain innocuous until activated. For instance, MBP-specific T cells present in 


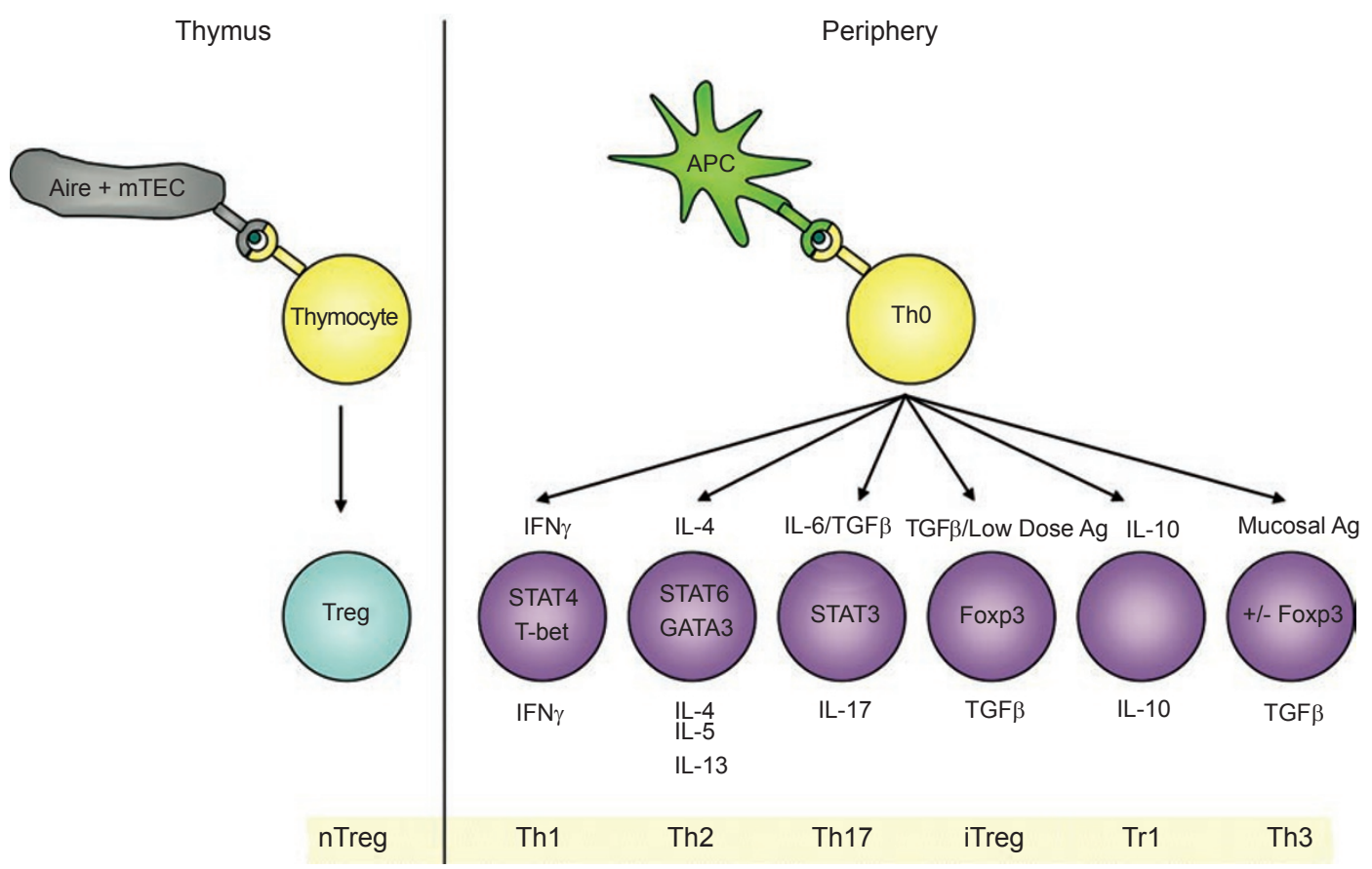

Figure 1 CD4+ T-cell lineage specification. Natural Tregs arise in the thymus as thymocytes interact with mTEC expressing tissuespecific antigens driven by the transcription factor AIRE. An increased affinity interaction between the peptide:MHC complex and the TCR along with secondary signals stimulates developing thymocytes to express Foxp3 and activates a gene expression profile driving natural Treg development. In the periphery, a number of different T-helper (Th) cell lineages exist. The cytokine milieu and transcription factors necessary to initiate their differentiation, as well as the soluble factors these cells characteristically express, are presented.

healthy humans and mice remain at bay until activated by immunization with MBP $[15,31]$. In TCR transgenic mice, where the frequency of MBP-specific T cells is increased, EAE can develop spontaneously [7]. In other words, if the frequency of auto-reactive $\mathrm{T}$ cells is high, immunization with antigen and adjuvant is not needed to activate those cells. This indicates that when a higher percentage of the CD4+ T-cell population is self-antigen-specific, the chances of auto-reactive T-cell activation are dramatically increased [12]. Overall, activation of these cells depends upon the number of self-reactive T cells, the amount and accessibility of self-antigen, the inflammatory cytokine environment at the time of antigen encounter, and the absence of suppressor cells to counteract the response.

Evidence to support that autoreactive CD4+ T cells can be actively suppressed was demonstrated in the early 1990s. In 1994, Lafaille et al. crossed MHC II (H-2 A $\left.{ }^{\text {u}}\right)$ restricted, MBP-specific TCR transgenic mice with RAG1-deficient mice [12]. RAG-1-deficient mice are incapable of forming endogenous BCR and TCR, and are thus B and $\mathrm{T}$ lymphocyte deficient. The cross of a TCR transgenic $(\mathrm{T} / \mathrm{R}+)$ to a $\mathrm{RAG}-1-/-$ mouse resulted in progeny $(\mathrm{T} / \mathrm{R}-)$ with T cells expressing only the MBP-specific TCR and no other lymphocytes. In their study, Lafaille et al. showed that $\mathrm{T} / \mathrm{R}$ - mice developed EAE $100 \%$ of the time when no other lymphocytes are present, while EAE only occurred $14 \%$ of the time in $\mathrm{T} / \mathrm{R}+$ mice with an otherwise normal immune system [12]. Thus, in the absence of other nontransgenic lymphocytes, MBP-specific CD4+ T cells induce spontaneous autoimmunity $100 \%$ of the time. However, the presence of the non-transgenic lymphocytes appears to have a protective effect that suppresses the potential of auto-reactive cells to cause spontaneous EAE. In another study, Kumar and Sercarz generated specific CD4+ T-cell clones from the lymph nodes of mice that had recovered from MBP-induced EAE, and showed that these CD4+ T-cell clones protected mice from MBP-induced EAE in adoptive transfer experiments [32]. In addition, Fowell and Mason showed that, in rats, normal non-autoimmune animals develop diabetes following a thymectomy, which renders them lymphopenic. Autoimmunity was completely prevented by replenishing syngeneic $T$ cells of a particular CD4+ subset shortly after treatment [33]. In total, these results suggest that a special subset of CD4+ T lympho- 
cytes created in the thymus is responsible for mediating the suppression of autoimmunity. Identification of the phenotype, development, and mechanism of suppression of these naturally occurring regulatory $\mathrm{CD} 4+\mathrm{T}$ cells will be the focus of this review.

\section{Identifying the suppressor cell: CD4+CD25+ Foxp3+ Tregs}

At this time, the role of a suppressive T-cell population of thymic origin was not a novel concept. Work from the late 1960s and early 1970s led by Gershon and Kondo introduced the concept of endogenous suppressor T cells with immunoregulatory properties [34]. This theory was largely refuted by the scientific community after a failure to clone the cell, and the inability to identify a lineage-specific marker. In 1994, after a lull in the field, CD4+ T-cell clones that were capable of suppressing MBP or PLP-induced EAE were reported [35]. As the theory of a suppressive factor was reignited, the history of the field stressed the importance of identifying unique markers which could be used to isolate the specific population of naturally arising 'regulatory' cells. Once identified, it could be determined whether removal of that subpopulation would contribute to a break in self-tolerance leading to autoimmunity, and whether its reconstitution could prevent the onset of disease [36].

\section{Regulatory T-cell surface markers}

Initial studies have shown that adoptive transfer of CD4+ T-cell populations depleted of $\mathrm{CD} 5^{\text {high }}$ or $\mathrm{CD} 45 \mathrm{RC}^{\text {low }} \mathrm{CD} 4+$ $\mathrm{T}$ cells to T-cell-deficient mice resulted in spontaneous autoimmune disease [37]. The use of CD45RC ${ }^{\text {low }}$ as a marker appeared to include the regulatory population; however, this marker is not unique to these cells, as its expression is downregulated on all CD4+ T cells after they have encountered antigen. An attempt to identify the specific CD4+ T cell type involved in suppression focused on the surface marker CD25, the IL-2 receptor $\alpha$-chain. The CD25+ cells belong to the $\mathrm{CD} 5^{\text {high }}, \mathrm{CD} 45 \mathrm{RC}^{\text {low }}$ population, and only $5-10 \%$ of peripheral CD4+ T cells (and less than $1 \%$ of CD8+ T cells) express CD25 [38]. Transfer of splenic cell suspensions depleted of CD4+ CD25+ T cells into Tcell-deficient recipients resulted in severe autoimmunity. Reconstitution of CD4+ CD25+ T cells within a short time after transfer prevented development of the disease [38]. Therefore, even though the normal immune system contains self-reactive CD4+ CD25- T cells capable of inducing autoimmunity, their activation is suppressed by a population of regulatory CD4 $+\mathrm{CD} 25+\mathrm{T}$ cells. Elimination of this population can break self-tolerance, leading to the onset of autoimmune disease.
The majority of CD4+ CD25+ Tregs constitutively express low levels of CTLA-4, a surface marker on T cells which outcompetes CD28 for binding to B7 (CD80/86) on APCs. The binding of CTLA-4 to B7 sends inhibitory signals to the T cell. CTLA-4 is also present on effector $\mathrm{T}$ cells, where it becomes active following costimulation and T-cell activation, as a mechanism to control lymphoproliferation. It has been suggested that this constitutively expressed marker plays a functional role in CD4+ CD25+ Treg-mediated suppression [39]. Another surface marker on $\mathrm{CD} 4+\mathrm{CD} 25+$ regulatory $\mathrm{T}$ cells is the glucocorticoidinduced TNF-receptor-related gene GITR. Constitutive, high-level expression of this molecule is considered a unique marker of Tregs as GITR is not present on naïve, conventional CD4+ T cells and is only upregulated on responder cells following TCR engagement [40]. The interaction of GITR on CD4+ CD25+ Tregs with its ligand (GITRL) on the surface of APCs abrogates suppression resulting in autoimmunity [41]. Evidence suggests that, in addition to attenuating Treg activity, engagement of GITR may also co-stimulate effector $\mathrm{T}$ cells, making them less susceptible to suppression [42]. The role of GITR-GITRL interactions in regulatory activity was recently reviewed by Shevach and Stephens [43].

\section{The master regulator: Foxp3}

While these cell surface markers, predominantly CD25, are useful for characterizing regulatory $\mathrm{T}$ cells in mice and humans, they are not expressed exclusively on Tregs. Expression of CD25 is upregulated upon activation of all T cells [44]. Therefore, the use of CD25 to differentiate regulatory T-cell populations depends upon a relative standard. CD4+ CD25+ Tregs are CD $4^{\text {low }} \mathrm{CD} 25^{\text {high }}$. They express CD25 constitutively and upon TCR stimulation the expression of this marker is higher and more persistent than on non-regulatory CD4+ T-cell populations $[44,45]$. The latter population of T cells, on the other hand, is CD25- prior to activation, and loses CD25 expression following stimulation. Due to its slightly subjective nature, CD25 is not a perfect marker for Treg identification. As such, discovery of a unique, lineage-specific marker for this subpopulation of cells was a substantial accomplishment for the field.

Identification of a unique Treg marker surfaced in an animal model of autoimmunity. Scurfy mice present lymphoproliferation, lymphocytic infiltration and multi-organ autoimmune disease [46]. Upon analysis, the disease was shown to be mediated by CD4+ T cells [47, 48]. In 2001, the mutation responsible for the scurfy phenotype in mice was identified in the gene encoding Foxp3 [49]. Mutation within the same gene in humans was determined to be the cause of the fatal autoimmune disorder IPEX [50]. Foxp3, named for its winged helix-forkhead DNA-binding domain, functions 
as a transcription factor. Full-length Foxp3 holds very high sequence homology across human, mouse, and rat [51]. In mice, this X-linked recessive disease appears to operate by a mechanism of dominant tolerance. Scurfy affects males, but not heterozygous females. Random X inactivation in heterozygous females results in a combination of cells with normal and defective Foxp3 [52]. There is no disease phenotype in these mice as the residual Foxp3-expressing cells dominantly control the population of self-reactive $\mathrm{T}$ cells. Foxp3-mutant scurfy mice are deficient in CD25+ CD4+ $\mathrm{T}$ cells, and the onset of autoimmunity can be prevented by expression of normal Foxp3+CD25+CD4+ Tregs [49, 53]. In 2003, Fontenot et al. generated conditional Foxp3null mice using homologous recombination, by creating mice carrying a floxed Foxp3 allele which can be crossed with a Cre-deleter transgenic strain to produce males with targeted deletion in Foxp3 [45]. As seen in Foxp3-mutant scurfy mice, the Foxp3-null mice exhibit CD4+ CD25+ Treg deficiency, resulting in a lethal, lymphoproliferative, autoimmune phenotype which can be rescued by transfer of regulatory T cells [45].

Functionally, Foxp3 + CD25+CD4+ regulatory T cells appear anergic in vitro: they do not proliferate or secrete IL-2 in response to TCR stimulation as their effector counterparts do [44, 45]. And yet, following crosslinking of the TCR, these cells are able to inhibit proliferation and cytokine production by those effectors [51]. Expression of Foxp 3 is necessary and sufficient for suppressor function of natural Tregs as Tregs from Foxp3+ mice maintain their characteristic behavior in vitro, being hyporesponsive to TCR stimulation themselves and capable of suppressing the proliferation of $\mathrm{CD} 4+\mathrm{CD} 25$ - effectors. In contrast, there is no suppressor activity in the $\mathrm{CD} 4+\mathrm{CD} 25+$ population from Foxp3- mice [45]. In addition, retroviral transduction of CD25- T cells with Foxp3 results in the functional conversion of these cells to Tregs with suppressive properties [54]. These cells exhibit decreased IL-2 production and increased expression of characteristic Treg surface markers such as CD25, CTLA-4, and GITR [53]. CD25- cells also acquire the ability to suppress effector T-cell proliferation in vitro and prevent autoimmunity in vivo following ectopic expression of Foxp3 [45].

Moreover, this transcription factor is unique to CD4+ CD25+ regulatory $\mathrm{T}$ cells. A comparison of mRNA and protein levels in CD25+ versus CD25- T cells showed that, while Foxp3 is highly expressed in CD25+ CD4+ CD8- peripheral $\mathrm{T}$ cells and thymocytes, it is present in low to undetectable levels in both naïve and activated CD4+ CD25- T cells, as well as in other T, B, NK, and NKT cells $[45,53,54]$. Overall, of the $15 \%$ of $\mathrm{T}$ cells express-

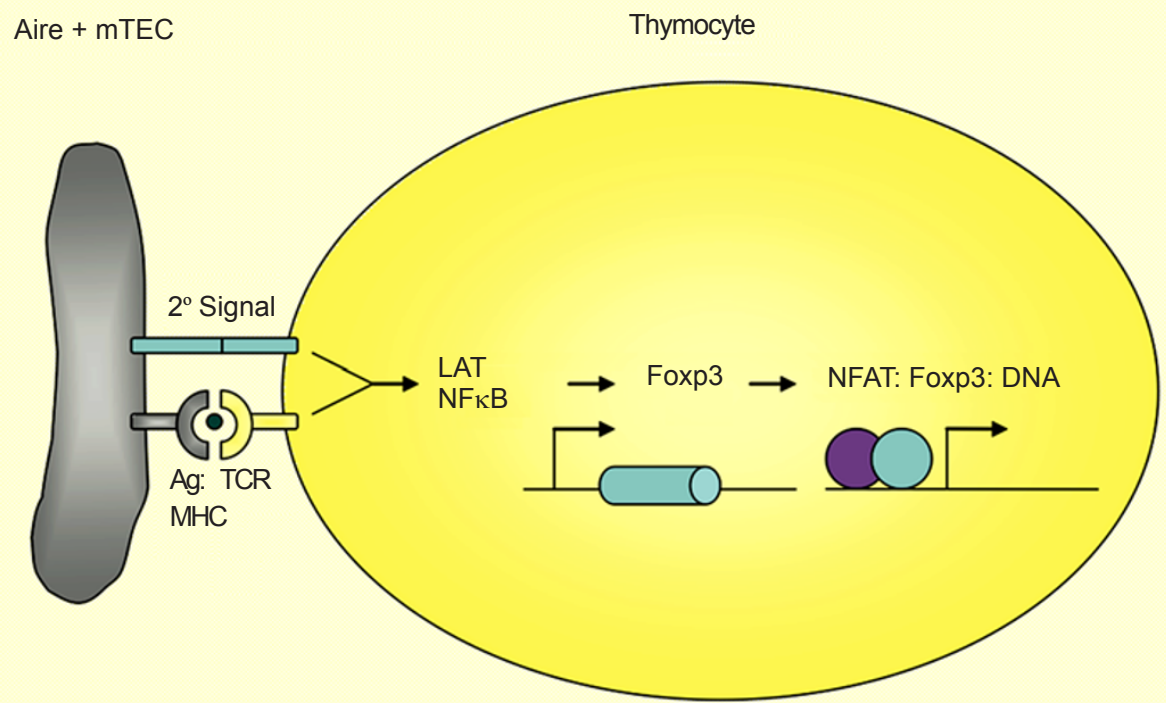

Figure 2 Lineage commitment of the natural Treg. In the thymus, developing thymocytes interact with Aire+ medullary thymic epithelial cells presenting tissue-specific self-antigens in the context of their MHC molecules. The combination of a high-affinity TCR interaction with the self-peptide:MHC complex along with secondary signals, such as CD28 engagement, direct Treg lineage commitment in the thymus. These interactions drive a Treg-specific signaling cascade which upregulates Foxp3 gene expression. The resultant Foxp3 protein can form a cooperative complex with NFAT on DNA, acting as a transcription factor to both repress genes involved in T-cell activation, such as IL-2 and IL-4, and activate those required for the Treg genetic program, such as CD25 and CTLA-4. 
ing Foxp3, 70-80\% are also CD4+ CD25+. Notably, these cells also constitutively express GITR at high levels [55]. Taken together, the transcription factor Foxp3 is specifically expressed in the CD4+CD25+ population of T cells, and is required for the generation of regulatory properties.

\section{Thymic development of natural Tregs}

Development of Foxp3 $+\mathrm{CD} 25+\mathrm{CD} 4+$ regulatory $\mathrm{T}$ cells occurs in the thymus (Figure 2). Removal of this organ by thymectomy in neonatal mice results in autoimmunity, which can be reversed by transferring peripheral CD25+ CD4+ Tregs [51]. CD25+ T cells appear to migrate to the periphery by day 3 , as thymectomy at day 3 , but not after, leads to autoimmunity. CD25+ cells can be isolated from the CD4+ thymocyte compartment of mice and humans and have suppressive activity in vitro and following adoptive transfer in vivo [56-58]. In addition to having the same in vitro and in vivo regulatory function as peripheral Tregs, CD25+CD4+ CD8-thymocytes are also similarly anergic and have the same surface molecule expression patterns: CTLA-4+ and GITR+.

\section{Role of TCR signaling}

The MHC class restriction of the thymocyte TCR dictates commitment to a T-cell lineage [6]. The role of TCR signaling in the development of CD4+ CD25+ Tregs was suggested by the observation that RAG-2 deficiency eliminates CD25+ thymocytes and T cells. Thus, TCRs endogenously rearranged in the thymus are required for the development of CD25+ Tregs in these mice. Furthermore, the proportion of Tregs that develop in TCR transgenic mice is increased in mice that are bred onto a strain expressing a transgene encoding the cognate ligand, speaking to the role of TCR-ligand interaction in Treg development [59-61]. The importance of TCR cross-linking in development is also supported by the observation that relative numbers of Tregs are reduced in mice with genetic defects in targets of downstream TCR signaling $[62,63]$. As discussed in the introduction, thymic development of Tregs requires an increased strength of TCR signaling somewhere above what is required for the positive selection of effector $\mathrm{T}$ cells, and below what will result in clonal deletion through negative selection [60].

Following TCR ligation, unique signaling pathways must be activated to initiate the transcriptional program required for Treg lineage commitment. Recent findings indicate that the signaling requirements needed to induce Treg development are distinct from those leading to the positive selection of other thymocytes. For instance, while a mutation in the tyrosine phosphorylation site in the Y136F variant of LAT (linker of activated T cells) results in a complete lack of Foxp3+ Tregs in both the thymus and periphery, there is only a partial defect in positive selection [64]. LAT is a transmembrane adaptor protein essential for T-cell activation and thymocyte development [65]. This study has indicated the involvement of LAT signaling in inducing Foxp3 expression and CD4+ CD25+ Treg development. Activation of the NF- $\kappa \mathrm{B}$ signaling pathway has also been implicated in Treg development as impairment of this pathway leads to a deficiency of Treg cells $[62,63]$. Recent reports question whether this effect is an indirect result of non-regulatory T cells becoming deficient in IL-2 production, which is required for the upregulation of CD25 and the peripheral maintenance of Tregs. The signaling pathways involved in thymic Treg development are reviewed in further detail by Liston and Rudensky [66].

It has also been suggested that Treg lineage commitment is not secured by the increased affinity of the TCR alone, but also relies on additional signals that remain poorly understood [67]. At the very least, secondary signaling events through other, antigen-independent surface molecules are likely to play a supporting role in thymic Treg development. The number of CD25+CD4+ CD8- thymocytes and Tregs is greatly reduced in CD28-, B7 (CD80/86)-, Il2-, Il2ra-, LFA-1-, or CD40-deficient mice [68]. Absence of these accessory molecules is likely to alter the interaction of developing Tregs with stromal cells of the thymic epithelium. Engagement of CD28 on the T-cell precursor to CD80/86, for example, is important for providing costimulation to the dominant TCR-mediated signal (Figure 2). Tregs developed in the absence of $\mathrm{CD} 28$ retain their repressive function, suggesting that, while CD28 engagement may enhance or alter it, this costimulatory factor is not required for Treg development [69].

\section{Role of Foxp 3}

The signaling pathways activated following an increased affinity TCR engagement in combination with secondary signals such as CD28 costimulation drive the expression of the lineage-specific marker, Foxp3 [45]. As a transcription factor, Foxp3 could then activate genes required for the unique Treg function and simultaneously repress those involved in other developmental pathways [70, 71] (Figure 2). It has been suggested that Foxp3 is needed for both development and function, as disrupting the Foxp3 gene blocks the development of natural Tregs or produces dysfunctional Tregs, the result of which is hyperactivation of auto-reactive $\mathrm{T}$ cells leading to autoimmunity [45, $53,54]$. Bone marrow chimera models demonstrate that Foxp3-deficient bone marrow cannot generate a CD25+ Treg population [45].

A recent paper, however, argues that, while the function of Tregs is Foxp3 dependent, development of this lineage 
may not entirely depend upon Foxp3 [72]. It is true that when Foxp3 is deficient, T cells lack regulatory function and mice develop autoimmunity. However, by labeling a non-functional version of Foxp3 with a GFP marker, Lin et al. have shown that pre-Tregs developing in the thymus retain certain intrinsic Treg qualities even though the Foxp3 protein they express is non-functional. Again, these cells failed to gain suppressor activity, yet they retain similar thymic development, cell surface phenotype, and a gene expression profile that is largely similar to that seen in Foxp3 expressing natural Tregs [72].

Overall, while there is no doubt that Foxp3 is a specific marker of developing Tregs, our understanding of the exact role that Foxp3 plays in directing development and lineage commitment remains incomplete. It appears that a certain increased avidity of TCR engagement (possibly in combination with other costimulatory interactions as discussed above) triggers a characteristic regulatory T-cell developmental pathway, marked by a certain pattern of thymic development and gene expression. Normally, the predominant gene induced by this signal is Foxp3, a transcription factor that regulates the gene expression profile of Tregs. The presence of this transcription factor is required for Tregs to acquire function, while certain components of the Treg phenotype may still be retained in its absence. As it seems, Foxp3 is not the only product of TCR engagement contributing to the acquisition of the natural Treg phenotype, though it is both necessary and sufficient for the generation of regulatory function in these cells.

The expression of Foxp3 and the subsequent development of regulatory $\mathrm{T}$ cells are also influenced by the development of the thymic epithelium itself. Thymic stromal cells not only provide the peptide-MHC complexes to engage immature TCRs but are also the source of secondary signals involved in dictating lineage commitment. Reports indicate that the development of Foxp3+ thymocytes is linked to that of the medullary thymic epithelium (mTEC). The majority of Foxp3 expressing thymocytes are localized to the medullary region of the thymus [73]. Another example of this link lies in the role of CD28. While Treg development requires the presence of CD28 [74], expression of the cognate ligand, B7 (CD80/86), is largely restricted to the mTEC [75]. Overall, it is believed that the additional signal required for Foxp3 expression, whether it is the expression of CD28 ligands or other cell surface molecules or cytokines, is localized to the mTEC.

\section{Peripheral maintenance of Tregs}

The expression of the IL-2R $\alpha$ chain (CD25) as a predominant marker of Treg function indicates that its ligand, interleukin 2 (IL-2), may be involved in the development, function, or maintenance of regulatory $\mathrm{T}$ cells. The necessity of IL-2 to Tregs was encouraged by the finding that IL-2- and IL-2R $\alpha / \beta$ chain deficiencies lead to an autoimmune phenotype in mice, and a reduction in the number of CD25+ CD4+ CD8- thymocytes and peripheral Tregs [76-78], both of which can be prevented by addition of IL-2 [79-81]. Neutralization of IL-2 with a monoclonal antibody also leads to the induction of autoimmunity in mice, a phenotype similar to that seen following Treg depletion. Foxp3+ CD25+ Tregs in the thymus and periphery are reduced in number and unable to proliferate in the absence of IL-2 [82]. Furthermore, the autoimmune phenotype in Balb/c mice following anti-IL-2 treatment can be adoptively transferred to nude mice. Co-transfer of CD25+ Tregs was able to prevent the onset of disease [82]. These results indicate that IL-2 is required for peripheral maintenance and activation of the regulatory T-cell population. Setoguchi et al. also described that activated nonregulatory $\mathrm{T}$ cells in the periphery, including autoreactive CD4+ cells, are the source of IL-2 [82]. Analysis of IL-2 mRNA and protein expression shows that its expression levels are high in CD4+ CD25- $/^{\text {low }}$ cells and low in the CD25+ Treg population.

As one would expect, the presence of IL-2 stimulates CD25 expression, increasing the number of CD25+ Tregs $[83,84]$. Conversely, an increase in Foxp3 expression within these cells is followed by a decrease in levels of IL-2 transcription as Foxp3 downregulates the IL-2 promoter [54]. Thus, while IL-2 appears to be required for the peripheral maintenance and activation of CD25+ Tregs, the expression of the Foxp3 transcription factor in these cells downregulates IL-2. A negative feedback control loop appears to be in place, in which effector T cells in the periphery secrete IL-2 to maintain and activate regulatory $\mathrm{T}$ cells, which downregulate their own IL-2 expression with Foxp3, and limit the expansion of those effectors. Once activated, regulatory $\mathrm{T}$ cells not only suppress the auto-reactive $\mathrm{T}$ cell population directly but also deprive them of the essential growth factor, IL-2.

It is important to remember that, while IL-2 deficiency significantly reduces the number of Foxp3+CD25+CD4+ Tregs, these cells are present in the absence of IL-2 [85]. A re-evaluation of the role of IL-2 has shown that this cytokine is dispensable for the development of Treg cells in the thymus, but essential for their peripheral maintenance [86]. In the thymus, it appears that signaling through the common $\gamma$ chain $(\gamma c, C D 132)$ rather than the IL-2R $\alpha$ chain is the crucial component in the development of Tregs [87]. Thus, in the absence of IL-2, these signals can be provided by other common $\gamma$-chain family cytokines. Evidence for the partially redundant role of IL-2 in the thymus has raised questions of whether the peripheral maintenance of Tregs 
can be supported in part by other $\gamma$-chain family cytokines as well. Yates et al. have recently confirmed that IL-2 maintains the regulatory phenotype of Tregs in vitro, preserving suppressive function and the expression of Foxp3, CD25, CTLA-4, and GITR, as well as preventing the apoptosis of Tregs [88]. However, other common gamma chain cytokines, such as IL-4, IL-7, and IL-15, were also able to maintain the maximal regulatory function of Tregs [88]. This study suggests that there is a degree of redundancy in the cytokines capable of maintaining peripheral Treg function in vitro. It will be important to understand the true role of IL-2 in vivo as well, as the peripheral requirements for Treg function may manifest similar redundancies as those seen in the thymic ontogeny of these cells.

\section{The function of Foxp3}

\section{Foxp3 as a transcription factor}

True to its role as a transcription factor, when Foxp 3 is expressed following TCR stimulation, it localizes to the nucleus and binds DNA to modulate gene expression as a transcriptional regulator [89]. Foxp3 has been shown to repress IL-2 and IL-4 gene transcription, and upregulate the expression of CD25 and CTLA-4 [54, 89, 90]. It was noticed that many of the genes regulated by Foxp3 are also target genes for the transcription factor NFAT [90]; NFAT upregulates IL-2, IL-4, CD25, and CTLA-4 [91-93]. NFAT includes four calcium-regulated transcription factors $[92,93]$, and in $\mathrm{T}$ cells it forms a strong cooperative complex with AP-1 proteins to upregulate expression of genes associated with T-cell activation [91]. While NFAT: AP1 complexes play a role in T-cell activation, NFAT can also upregulate negative regulators of $\mathrm{T}$ cell signaling in an AP-1-independent manner, contributing to T-cell anergy $[94,95]$. The assumption that Foxp3 and NFAT interact in some way was supported by the identification of forkhead binding domains adjacent to NFAT transcription factor binding sites in the promoters of several cytokine genes (including IL-2, IL-4, and TNF) [89, 96]. Several mechanisms of interaction have been suggested: (1) Foxp3 and NFAT compete for DNA binding [89]; (2) Foxp3 sequesters NFAT, preventing it from binding DNA to activate $\mathrm{T}$ cells [97]; and (3) a cooperative complex forms between NFAT and Foxp3 [90]. In 2006, Wu et al. provided convincing evidence for the latter theory, showing that Foxp3 inhibits the formation of nuclear NFAT:AP1:DNA complexes by forming an NFAT:Foxp3:DNA complex [90] (Figure 2). Chromatin immunoprecipitation (ChIP) experiments confirmed that both transcription factors could occupy the IL-2, CTLA-4, and CD25 promoters, and that NFAT binding at these sites was substantially increased in Foxp3-expressing cells, suggesting that Foxp3 expression stabilizes NFAT promoter binding. Structure-based mutations of Foxp3, disrupting its interaction with NFAT, were shown to decrease its ability to repress IL-2. Mutations in this interface also interfered with the ability of retrovirally transduced Foxp3 to upregulate CTLA-4 and to a lesser extent CD25, CD103 and GITR expression. These mutations go on to impair the regulatory function of Foxp3 expressing Tregs, which become incapable of preventing autoimmunity [90]. Thus, the transcriptional role of Foxp3 in developing suppressor function depends crucially on the integrity of the Foxp3: NFAT interface, suggesting that a cooperative complex is formed between NFAT, Foxp3, and DNA.

\section{Targets of Foxp 3}

While it appears to regulate transcription through cooperative interaction with other transcription factors such as NFAT, the transcriptional program of Foxp3 remains largely undefined. Two recent papers have supplied more evidence for the precise function of Foxp3 by elucidating target genes of this transcription factor in a genome-wide approach [70, 71].

Marson et al. used ChIP combined with DNA microarrays to identify genes occupied by the transcription factor Foxp3 [71]. Foxp3- CD4+ T-cell hybridomas with and without transduction of FLAG-tagged Foxp3 were used to compare the specific effect of this transcription factor on gene expression. They discovered that the promoters of 1119 genes are direct targets of Foxp3 binding. Included in this list are promoters for IL-2, CD25, and GITR. Consensus forkhead binding motifs were located at each of these genomic loci, and neighboring sites were enriched for NFAT-binding sequence motifs, supporting the idea of cooperative complex formation between the two transcription factors. By comparing the list of genes occupied by Foxp3 to the biological pathways in which those genes are activated, it appears that Foxp3's targets are most strongly associated with the TCR signaling and activation pathway. In order to determine whether Foxp3 binding truly affects expression of these target genes, microarray expression profiling was pursued to identify genes that were differentially expressed in Foxp3+ versus Foxp3- hybridomas. In unstimulated cells, there were few differences in the gene expression profile with or without Foxp3. However, stimulated cells express a number of genes differentially, depending on the presence of this transcription factor. To be exact, they identified 125 differentially expressed genes in Foxp3+ versus Foxp3- hybridoma cells. In stimulated Foxp3+ cells, Foxp3 binding was predominantly associated with downregulation of target genes that are normally upregulated during TCR stimulation and T-cell activation. The majority of the targets regulated in the hybridoma model are similarly regulated following stimulation of 
primary cells ex vivo, where the overall activation status is heterogeneous. The few differences in gene expression noted may be explained by the presence of transcriptional cofactors in ex vivo cultured cells. Overall, Marson et al. determined that the targets of Foxp3 binding are largely genes involved in TCR signaling and stimulation, and, concurrent with the role of Foxp3 in Tregs, binding of this transcription factor to its target genes appears to downregulate their expression [71]. According to these findings, the main transcriptional role of Foxp3 is to suppress genes involved in T-cell activation.

A slightly different approach was taken by Zheng et al., who used ChIP in combination with a genome tiling array to identify over 700 genes which are targets for Foxp3 binding [70]. They first demonstrated that the frequency of Foxp3 binding progressively decreases with increasing distance from the transcription start site. Preferential positioning of Foxp3-binding sites in the proximity of promoters or within the first introns provided evidence for the role of Foxp3 in classical transcriptional regulation. Gene ontology analysis revealed that Foxp3 targets are again enriched for genes involved in TCR signaling. Targets identified in this study also include genes involved in cell communication and transcriptional regulation. To confirm that Foxp3 binding played a functional role, this group also looked for genes whose expression was altered in a Foxp3-dependent manner. They reported that many Foxp3-bound genes were differentially expressed in regulatory $\mathrm{T}$ cells relative to naïve or activated effectors. As demonstrated by Marson et al., following stimulation with PMA and ionomycin, there was an increase of Foxp3 occupancy of the IL-2 promoter in regulatory $\mathrm{T}$ cells, supporting their role in IL-2 repression $[70,71]$. In addition, targets subjected to Foxp3-dependent modulation included genes encoding characteristic Treg surface markers, such as CD25 and CTLA-4. Other differentially expressed targets of Foxp3 include a number of transcription factor-encoding genes. Interestingly however, $35 \%$ of Foxp3-bound genes were upregulated in Treg cells in the thymus and $6 \%$ in the periphery, while the proportion of those genes being downregulated was smaller in both locations. This finding challenges the accepted role of Foxp3 as a predominant repressor. Zheng et al. also analyzed Foxp3 binding sites for the presence of histone modifications to determine whether they play a mechanistic role in Foxp3-mediated gene regulation. Permissive histone modifications were highly prevalent in Foxp3-upregulated genes, but rare in those genes repressed by Foxp3. In addition, genes repressed by Foxp3 were enriched for inhibitory H3 modifications. This supports the idea that histone modifications are involved in Foxp3-mediated gene regulation [70]. Overall, Foxp3 appears to function as a transcriptional activator and repressor, which not only directly regulates expression of the characteristic Treg surface molecules and target genes involved in TCR signaling, but also indirectly mediates their development by targeting a network of transcription factors and epigenetic chromatin modifications to further control gene expression.

\section{Mechanism of suppression}

Identification of Treg-specific markers allowing for enrichment in cell culture has contributed to the development of in vitro systems to study Treg-mediated suppression, by analyzing the proliferation of non-regulatory $\mathrm{T}$ cells either alone or in co-culture with Tregs [98]. The ability to study these cells in vitro has been helpful in elucidating their functional characteristics, although the mechanisms of tolerance are still largely undefined. What has become apparent is that Tregs appear to operate by multiple mechanisms of suppression, ranging from indirect suppression through secretion of soluble factors such as cytokines to direct suppression through binding of cell surface molecules [99] (Figure 3). While suppressor function requires Tregs to be activated through their TCR, these cells display distinct functional properties in vitro versus in vivo [98].

\section{Membrane-associated mechanisms of suppression (contact dependent)}

Suppression in vitro appears to be contact dependent as it does not occur when cells are separated by a permeable membrane [98]. It is also not affected by lack of soluble factors such as TGF $\beta$ or IL-10, as Tregs isolated from mice with deletions in these genes retain their suppressive activity in vitro [98-100]. Antibodies to TGF $\beta$, on the other hand, do inhibit suppressor function in vivo, suggesting a contact-dependent mechanism involving surface-bound TGF $\beta$ [101]. Because Tregs constitutively express the inhibitory surface molecule CTLA-4, there has been interest in determining whether binding of this molecule to B7 (CD80/86) on APC or T cells may play a role in suppression. Indeed, treatment with monoclonal antibody against CTLA-4 results in an autoimmune phenotype similar to the one seen after CD4+CD25+ Treg depletion [102]. One proposed mechanism involves the interaction of $\mathrm{B} 7$ (CD80/86) on APC or activated T cells with CTLA-4 constitutively expressed on Tregs, sending an inhibitory signal to the APC or T cell [103] (Figure 3). This theory is supported by the finding that suppression of $\mathrm{T}$ cells not expressing CD80/86 is reduced compared to the suppression of wildtype T cells in vitro [103]. There is contradictory evidence on this matter, however, as Tregs from CTLA-4-deficient mice have the same suppressive activity in vitro as those from normal mice [39, 102]. Furthermore, this contact-dependent mechanism does not appear to operate indirectly 


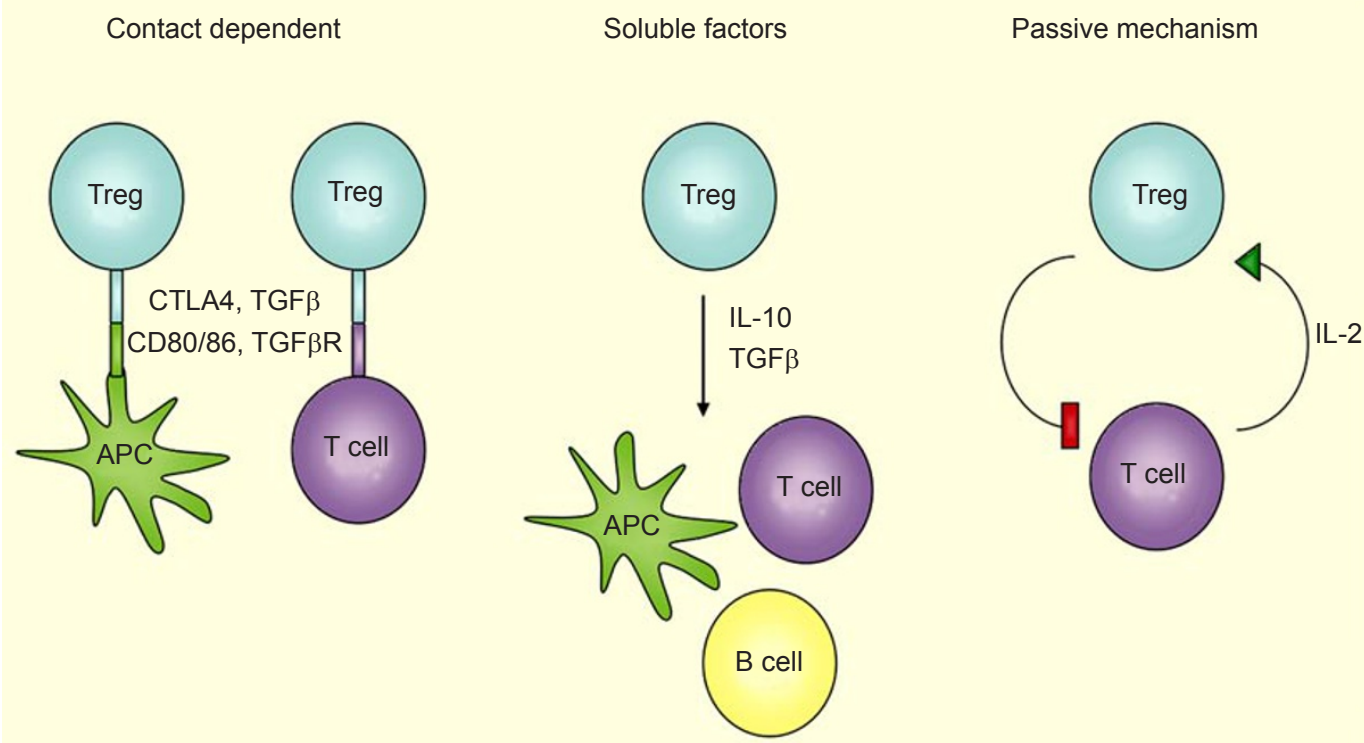

Figure 3 Mechanisms of regulatory T-cell suppression. Treg-mediated suppression is suspected to be either contact-dependent, driven by soluble factors, or fueled by IL-2 in a passive manner. Contact-dependent suppression involves interaction of CTLA-4 or TGF $\beta$ on the Treg with cognate receptors on the target cell, B7 (CD80/86) and TGFßRII, respectively. This direct physical interaction may result in suppression of the target cell or death of that cell through Granzyme B secretion. Bystander suppression can occur by the secretion of soluble factors, such as the cytokines IL-10 and TGF $\beta$. In the passive mechanism, non-regulatory T cells produce IL-2 upon activation, fueling the expansion of Tregs and their acquisition of suppressor function. As Tregs take up IL-2, the cytokine is also sequestered from the non-regulatory T-cell population, depriving effector T cells of this essential growth factor.

through APCs as suppression persists in APC-free culture conditions [98]. If in vitro suppression is contact dependent, direct killing by the release of cytolytic molecules such as perforin and granzyme is also a possibility. Grossman et al. have shown that Tregs activated by a combination of CD3 and CD46 can express granzyme and kill activated T cells in a Fas-independent manner [104].

\section{Soluble factors: cytokine-driven suppression}

The observation that suppression is prevented by a permeable membrane does not eliminate the possibility of soluble mediators working over a short distance. Furthermore, the finding that suppressors and their targets can co-exist for long periods of time without an increase in the rate of apoptosis in target cells suggests that direct killing through cytotoxicity is not the major mechanism of suppression in vivo $[105,106]$. Suppression also appears to be reversible as separation of previously co-existing suppressor and target cells shows that CD4+CD25- cells can proliferate and secrete IL-2 following antigenic stimulation when the Tregs are removed $[105,106]$. In fact, if the effector $\mathrm{T}$ cells were committed to a certain lineage prior to suppression, that commitment is retained following the removal of suppressor cells [105]. These data argue that
Tregs mediate reversible suppression through the release of soluble factors (Figure 3 ).

Both IL-10 and TGF- $\beta$ have been linked to suppression in vivo, although specific mechanisms have not been confirmed [98, 99, 107, 108]. The role for IL-10, for instance, is ambiguous; Tregs require secretion of this cytokine in order to suppress some autoimmune diseases such as colitis, while it is not required for suppression of others (e.g., autoimmune gastritis) [98, 105, 107-109]. The story in vivo is complicated by the fact that several classes of regulatory $\mathrm{T}$ cells exist in the periphery, all of which may utilize different mechanisms of suppression, and some of which do rely on soluble factors. It is possible that secretion of soluble factors also contributes to suppression by natural Tregs, although more conclusive evidence is required.

A passive mechanism of suppression: competition for IL-2

As mentioned above, IL-2- or IL-2R-deficient mice lack CD25+ Tregs and develop severe autoimmune diseases, which can be prevented by transferring CD4+ CD25+ regulatory T cells in vivo $[79,110]$. This suggests that IL-2 plays a role in Treg maintenance and function $[81,84,111]$. Due to the importance of the growth factor IL-2, a passive mechanism has been proposed in which Tregs mediate sup- 
pression by sequestering the IL-2 produced by non-regulatory T cells: IL-2 fuels Treg expansion and suppressive function, while effector $T$ cells suffer in the absence of this essential growth factor [105, 112] (Figure 3).

There are likely multiple, redundant mechanisms of suppression by natural Tregs, which vary depending on the tissue and model of inflammation being studied. Different tissues have different microenvironments and concentrations of antigen to fuel suppressor activity in various ways. The mechanism of action may take place indirectly over a short distance by soluble mediators such as cytokines, or through direct cell contact of suppressors and either APC intermediates or the effector T-cell targets themselves. Regardless of the mechanism, suppression requires the ability of Tregs to localize with APC and ligand for priming by TCR stimulation, as well as the ability to migrate to inflamed tissues to find their targets $[99,113]$. Overall, the degree of Treg-mediated suppression is dependent on the frequency of CD25+CD4+ T cells, access to sufficient concentration of antigen presented by APC, and localization with effector T-cell targets. The cytokine environment required to support Treg growth, maintenance, and activation of suppressor function in the periphery at the time of antigen encounter is critical as well.

\section{Induced Tregs and other regulatory populations}

Thymic development is not the only means of generating regulatory $\mathrm{T}$ cells, and naturally occurring Tregs are not the only subset of $\mathrm{T}$ cells exhibiting suppressor function [114]. The focus of this review has been on the thymically derived class of naturally occurring suppressors, referred to as nTregs. However, $\mathrm{T}$ cells exhibiting regulatory function can develop in the periphery as well (Figure 1). Naïve CD4+ CD25- Foxp3-T cells in the periphery can be converted into Foxp3+ CD25+ CD4+ Tregs with suppressor function following TCR stimulation in the presence of low-dose peptide antigen or TGF $\beta$ [115-117]. These 'induced' or 'adaptive' Tregs mediate suppressor activity through secretion of TGF $\beta$ or IL-10 [115]. Interestingly, while TGF $\beta$ in the steady state fuels differentiation of induced Tregs which control autoimmunity in the periphery, the presence of TGF $\beta$ in combination with IL- 6 following inflammation drives development of Th17 cells, which themselves induce autoimmune diseases [23]. This fine line in the differentiation of two counteractive T-cell subsets illustrates how precisely balanced the immune system must remain in order to generate the necessary response. Another regulatory subset, the antigen-specific ' $\operatorname{Tr} 1$ ' cells, can be generated by culturing CD4+ T cells with antigen in the presence of IL-10 [118]. As reviewed by Shevach, antigen-specific Tr1 cells can also be induced by IL-10 in vivo in order to control the inflammatory response to infectious pathogens [114]. Notably, these cells do not express Foxp3. A mucosal type of Treg, designated as Th3 cells, can be induced by food antigens and play important roles in preventing food hypersensitivity [35]. These cells can be either Foxp3+ or Foxp3 [119]. They can also be induced to suppress autoimmune diseases following oral administration of the corresponding self-antigens. And if this were not enough to keep the autoreactive effectors at bay, data suggest that certain populations of CD8+ and CD4- CD8- double-negative regulatory $\mathrm{T}$ cells exist as well [114].

\section{Prospects}

The suppressor T-cell field has come a long way since the 1980 s, when it seemed that the scientific community had all but given up on the idea that a class of T cells with suppressor function could truly exist. In the past several decades researchers have made significant progress: from cloning suppressor cells, to identifying specific markers, delineating regulatory subtypes, and understanding their development, phenotype, peripheral maintenance, and function. Despite all of the advances in the field, much remains to be discovered. The contributions of Marson et al. and Zheng et al. in identifying Foxp3 target genes will certainly fuel interest in Foxp3-mediated gene expression and its role in regulatory T-cell development. Details of thymic lineage commitment, including the role of other transcription factors with cooperative or independent roles in gene regulation, are of interest. Accessory signaling molecules and the upstream genetic program directing expression of Foxp3 following TCR stimulation both in the thymus and in the periphery also remain unknown. A greater understanding of the precise delineations between subclasses of T cells that possess regulatory function is certainly in our future, as is the search for their exact mechanisms of suppression both in vitro and in vivo. With a renewed interest in the existence of this unique population of cells, in addition to their potential for treating autoimmunity and regulating the immune response to tumors or infectious pathogens, there is little doubt that the field will continue to answer these questions in the decades to come.

\section{References}

1 von Boehmer H, isielow P. Self-nonself discrimination by T cells. Science 1990; 248:1369-1373.

2 Oettinger MA, Schatz DG, Gorka C, Baltimore D. RAG-1 and RAG-2, adjacent genes that synergistically activate V(D)J recombination. Science 1990; 248:1517-1523.

3 Rocha B, von Boehmer H. Peripheral selection of the T cell repertoire. Science 1991; 251:1225-1228. 
4 Kisielow P, Teh HS, Bluthmann H, von Boehmer H. Positive selection of antigen-specific $\mathrm{T}$ cells in thymus by restricting MHC molecules. Nature 1988; 335:730-733.

5 Anderson MS, Venanzi ES, Klein L, et al. Projection of an immunological self shadow within the thymus by the aire protein. Science 2002; 298:1395-1401.

6 Goldrath AW, Bevan MJ. Selecting and maintaining a diverse T-cell repertoire. Nature 1999; 402:255-262.

7 Goverman J, Woods A, Larson L, et al. Transgenic mice that express a myelin basic protein-specific T cell receptor develop spontaneous autoimmunity. Cell 1993; 72:551-560.

8 Lassmann H, Bruck W, Lucchinetti CF. The immunopathology of multiple sclerosis: an overview. Brain Pathol 2007; 17:210218.

9 Tabira T, Sakai K. Demyelination induced by T cell lines and clones specific for myelin basic protein in mice. Lab Invest 1987; 56:518-525.

10 Jiang H, Zhang SI, Pernis B. Role of CD8+ T cells in murine experimental allergic encephalomyelitis. Science 1992; 256:12131215.

11 Koh DR, Fung-Leung WP, Ho A, et al. Less mortality but more relapses in experimental allergic encephalomyelitis in CD8-/mice. Science 1992; 256:1210-1213.

12 Lafaille JJ, Nagashima K, Katsuki M, Tonegawa S. High incidence of spontaneous autoimmune encephalomyelitis in immunodeficient anti-myelin basic protein $\mathrm{T}$ cell receptor transgenic mice. Cell 1994; 78:399-408.

13 Traugott U. Multiple sclerosis: relevance of class I and class II MHC-expressing cells to lesion development. J Neuroimmunol 1987; 16:283-302.

14 Todd JA, Acha-Orbea H, Bell JI, et al. A molecular basis for MHC class II-associated autoimmunity. Science 1988; 240:10031009.

15 Ota K, Matsui M, Milford EL, et al. T-cell recognition of an immunodominant myelin basic protein epitope in multiple sclerosis. Nature 1990; 346:183-187.

16 Abbas AK, Murphy KM, Sher A. Functional diversity of helper T lymphocytes. Nature 1996; 383:787-793.

17 Bettelli E, Oukka M, Kuchroo VK. T(H)-17 cells in the circle of immunity and autoimmunity. Nat Immunol 2007; 8:345-350.

18 Mosmann TR, Coffman RL. TH1 and TH2 cells: different patterns of lymphokine secretion lead to different functional properties. Annu Rev Immunol 1989; 7:145-173.

19 Khoury SJ, Hancock WW, Weiner HL. Oral tolerance to myelin basic protein and natural recovery from experimental autoimmune encephalomyelitis are associated with downregulation of inflammatory cytokines and differential upregulation of transforming growth factor beta, interleukin 4, and prostaglandin E expression in the brain. J Exp Med 1992; 176:1355-1364.

20 Ando DG, Clayton J, Kono D, Urban JL, Sercarz EE. Encephalitogenic $\mathrm{T}$ cells in the B10.PL model of experimental allergic encephalomyelitis (EAE) are of the Th-1 lymphokine subtype. Cell Immunol 1989; 124:132-143.

21 Bettelli E, Sullivan B, Szabo SJ, et al. Loss of T-bet, but not STAT1, prevents the development of experimental autoimmune encephalomyelitis. J Exp Med 2004; 200:79-87.

22 Chitnis T, Najafian N, Benou C, et al. Effect of targeted disruption of STAT4 and STAT6 on the induction of experimental autoimmune encephalomyelitis. J Clin Invest 2001; 108:739- 747.
23 Bettelli E, Carrier Y, Gao W, et al. Reciprocal developmental pathways for the generation of pathogenic effector TH17 and regulatory T cells. Nature 2006; 441:235-238.

24 Park H, Li Z, Yang XO, et al. A distinct lineage of CD4 T cells regulates tissue inflammation by producing interleukin 17 . Nat Immunol 2005; 6:1133-1141.

25 Nardelli DT, Burchill MA, England DM, et al. Association of CD4+ CD25+ $\mathrm{T}$ cells with prevention of severe destructive arthritis in Borrelia burgdorferi-vaccinated and challenged gamma interferon-deficient mice treated with anti-interleukin-17 antibody. Clin Diagn Lab Immunol 2004; 11:1075-1084.

26 Tran EH, Prince EN, Owens T. IFN-gamma shapes immune invasion of the central nervous system via regulation of chemokines. J Immunol 2000; 164:2759-2768.

27 Willenborg DO, Fordham S, Bernard CC, Cowden WB, Ramshaw IA. IFN-gamma plays a critical down-regulatory role in the induction and effector phase of myelin oligodendrocyte glycoprotein-induced autoimmune encephalomyelitis. J Immunol 1996; 157:3223-3227.

28 Hofstetter HH, Ibrahim SM, Koczan D, et al. Therapeutic efficacy of IL-17 neutralization in murine experimental autoimmune encephalomyelitis. Cell Immunol 2005; 237:123-130.

29 Matusevicius D, Kivisakk P, He B, et al. Interleukin-17 mRNA expression in blood and CSF mononuclear cells is augmented in multiple sclerosis. Mult Scler 1999; 5:101-104.

30 Korn T, Reddy J, Gao W, et al. Myelin-specific regulatory T cells accumulate in the CNS but fail to control autoimmune inflammation. Nat Med 2007; 13:423-431.

31 Fritz RB, Chou CH, McFarlin DE. Induction of experimental allergic encephalomyelitis in PL/J and (SJL/J x PL/J)F 1 mice by myelin basic protein and its peptides: localization of a second encephalitogenic determinant. J Immunol 1983; 130:191-194.

32 Kumar V, Sercarz EE. The involvement of T cell receptor peptide-specific regulatory CD4+ T cells in recovery from antigeninduced autoimmune disease. J Exp Med 1993; 178:909-916.

33 Fowell D, Mason D. Evidence that the T cell repertoire of normal rats contains cells with the potential to cause diabetes. Characterization of the CD4+ T cell subset that inhibits this autoimmune potential. J Exp Med 1993; 177:627-636.

34 Gershon RK, Kondo K. Infectious immunological tolerance. Immunology 1971; 21:903-914.

35 Chen Y, Kuchroo VK, Inobe J, Hafler DA, Weiner HL. Regulatory $\mathrm{T}$ cell clones induced by oral tolerance: suppression of autoimmune encephalomyelitis. Science 1994; 265:1237-1240.

36 Sakaguchi S. Regulatory T cells: key controllers of immunologic self-tolerance. Cell 2000; 101:455-458.

37 Sakaguchi S, Fukuma K, Kuribayashi K, Masuda T. Organ-specific autoimmune diseases induced in mice by elimination of $\mathrm{T}$ cell subset. I. Evidence for the active participation of T cells in natural self-tolerance; deficit of a $\mathrm{T}$ cell subset as a possible cause of autoimmune disease. J Exp Med 1985; 161:72-87.

38 Sakaguchi S, Sakaguchi N, Asano M, Itoh M, Toda M. Immunologic self-tolerance maintained by activated $\mathrm{T}$ cells expressing IL-2 receptor alpha-chains (CD25). Breakdown of a single mechanism of self-tolerance causes various autoimmune diseases. J Immunol 1995; 155:1151-1164.

39 Tang Q, Boden EK, Henriksen KJ, et al. Distinct roles of CTLA-4 and TGF-beta in $\mathrm{CD} 4+\mathrm{CD} 25+$ regulatory $\mathrm{T}$ cell function. Eur $\mathrm{J}$ Immunol 2004; 34:2996-3005. 
40 Zwar TD, van Driel IR, Gleeson PA. Guarding the immune system: suppression of autoimmunity by CD4+CD25+ immunoregulatory T cells. Immunol Cell Biol 2006; 84:487-501.

41 Shimizu J, Yamazaki S, Takahashi T, Ishida Y, Sakaguchi S. Stimulation of CD25(+)CD4(+) regulatory T cells through GITR breaks immunological self-tolerance. Nat Immunol 2002; 3:135142.

42 Stephens GL, McHugh RS, Whitters MJ, et al. Engagement of glucocorticoid-induced TNFR family-related receptor on effector $\mathrm{T}$ cells by its ligand mediates resistance to suppression by CD4+CD25+ T cells. J Immunol 2004; 173:5008-5020.

43 Shevach EM, Stephens GL. The GITR-GITRL interaction: costimulation or contrasuppression of regulatory activity? Nat Rev Immunol 2006; 6:613-618.

44 Kuniyasu Y, Takahashi T, Itoh M, et al. Naturally anergic and suppressive CD25(+)CD4(+) T cells as a functionally and phenotypically distinct immunoregulatory $\mathrm{T}$ cell subpopulation. Int Immunol 2000; 12:1145-1155.

45 Fontenot JD, Gavin MA, Rudensky AY. Foxp3 programs the development and function of CD4+CD25+ regulatory T cells. Nat Immunol 2003; 4:330-336.

46 Godfrey VL, Wilkinson JE, Russell LB. X-linked lymphoreticular disease in the scurfy (sf) mutant mouse. Am J Pathol 1991; 138:1379-1387.

47 Godfrey VL, Wilkinson JE, Rinchik EM, Russell LB. Fatal lymphoreticular disease in the scurfy (sf) mouse requires T cells that mature in a sf thymic environment: potential model for thymic education. Proc Natl Acad Sci USA 1991; 88:5528-5532.

48 Blair PJ, Bultman SJ, Haas JC, et al. CD4+CD8- T cells are the effector cells in disease pathogenesis in the scurfy (sf) mouse. J Immunol 1994; 153:3764-3774.

49 Brunkow ME, Jeffery EW, Hjerrild KA, et al. Disruption of a new forkhead/winged-helix protein, scurfin, results in the fatal lymphoproliferative disorder of the scurfy mouse. Nat Genet 2001; 27:68-73.

50 Bennett CL, Christie J, Ramsdell F, et al. The immune dysregulation, polyendocrinopathy, enteropathy, X-linked syndrome (IPEX) is caused by mutations of FOXP3. Nat Genet 2001; 27:20-21.

51 Fontenot JD, Rudensky AY. A well adapted regulatory contrivance: regulatory $\mathrm{T}$ cell development and the forkhead family transcription factor Foxp3. Nat Immunol 2005; 6:331-337.

52 Tommasini A, Ferrari S, Moratto D, et al. X-chromosome inactivation analysis in a female carrier of FOXP3 mutation. Clin Exp Immunol 2002; 130:127-130.

53 Khattri R, Cox T, Yasayko SA, Ramsdell F. An essential role for Scurfin in CD4+CD25+ T regulatory cells. Nat Immunol 2003; 4:337-342.

54 Hori S, Nomura T, Sakaguchi S. Control of regulatory T cell development by the transcription factor Foxp3. Science 2003; 299:1057-1061.

55 Ono M, Shimizu J, Miyachi Y, Sakaguchi S. Control of autoimmune myocarditis and multiorgan inflammation by glucocorticoid-induced TNF receptor family-related protein(high), Foxp3expressing CD25+ and CD25- regulatory T cells. J Immunol 2006; 176:4748-4756.

56 Stephens LA, Mason D. CD25 is a marker for CD4+ thymocytes that prevent autoimmune diabetes in rats, but peripheral $\mathrm{T}$ cells with this function are found in both CD25+ and CD25- subpopu- lations. J Immunol 2000; 165:3105-3110.

57 Papiernik M, de Moraes ML, Pontoux C, Vasseur F, Penit C. Regulatory CD4 T cells: expression of IL-2R alpha chain, resistance to clonal deletion and IL-2 dependency. Int Immunol 1998; 10:371-378.

58 Itoh M, Takahashi T, Sakaguchi N, et al. Thymus and autoimmunity: production of CD25+CD4+ naturally anergic and suppressive $\mathrm{T}$ cells as a key function of the thymus in maintaining immunologic self-tolerance. J Immunol 1999; 162:5317-5326.

59 Walker LS, Chodos A, Eggena M, Dooms H, Abbas AK. Antigen-dependent proliferation of CD4+ CD25+ regulatory T cells in vivo. J Exp Med 2003; 198:249-258.

60 Jordan MS, Boesteanu A, Reed AJ, et al. Thymic selection of CD4+CD25+ regulatory $\mathrm{T}$ cells induced by an agonist self-peptide. Nat Immunol 2001; 2:301-306.

61 Apostolou I, Sarukhan A, Klein L, von Boehmer H. Origin of regulatory $\mathrm{T}$ cells with known specificity for antigen. Nat Immunol 2002; 3:756-763.

62 Schmidt-Supprian M, Tian J, Grant EP, et al. Differential dependence of CD4+CD25+ regulatory and natural killer-like T cells on signals leading to NF-kappaB activation. Proc Natl Acad Sci USA 2004; 101:4566-4571.

63 Schmidt-Supprian M, Courtois G, Tian J, et al. Mature T cells depend on signaling through the IKK complex. Immunity 2003; 19:377-389.

64 Koonpaew S, Shen S, Flowers L, Zhang W. LAT-mediated signaling in CD4+CD25+ regulatory T cell development. J Exp Med 2006; 203:119-129.

65 Zhang W, Sommers CL, Burshtyn DN, et al. Essential role of LAT in T cell development. Immunity 1999; 10:323-332.

66 Liston A, Rudensky AY. Thymic development and peripheral homeostasis of regulatory T cells. Curr Opin Immunol 2007; 19:176-185.

67 Viret C, Janeway CA Jr. Self-specific MHC class II-restricted CD4-CD8- T cells that escape deletion and lack regulatory activity. J Immunol 2003; 170:201-209.

68 Sakaguchi S, Ono M, Setoguchi R, et al. Foxp3+ CD25+CD4+ natural regulatory $\mathrm{T}$ cells in dominant self-tolerance and autoimmune disease. Immunol Rev 2006; 212:8-27.

69 Salomon B, Lenschow DJ, Rhee L, et al. B7/CD28 costimulation is essential for the homeostasis of the CD4+CD25+ immunoregulatory $\mathrm{T}$ cells that control autoimmune diabetes. Immunity 2000; 12:431-440.

70 Zheng Y, Josefowicz SZ, Kas A, et al. Genome-wide analysis of Foxp3 target genes in developing and mature regulatory T cells. Nature 2007; 445:936-940.

71 Marson A, Kretschmer K, Frampton GM, et al. Foxp3 occupancy and regulation of key target genes during T-cell stimulation. Nature 2007; 445:931-935.

72 Lin W, Haribhai D, Relland LM, et al. Regulatory T cell development in the absence of functional Foxp3. Nat Immunol 2007; 8:359-368.

73 Fontenot JD, Rasmussen JP, Williams LM, et al. Regulatory T cell lineage specification by the forkhead transcription factor foxp3. Immunity $2005 ; 22: 329-341$.

74 Tai X, Cowan M, Feigenbaum L, Singer A. CD28 costimulation of developing thymocytes induces Foxp3 expression and regulatory $\mathrm{T}$ cell differentiation independently of interleukin 2 . Nat Immunol 2005; 6:152-162. 
75 Fontenot JD, Dooley JL, Farr AG, Rudensky AY. Developmental regulation of Foxp3 expression during ontogeny. J Exp Med 2005; 202:901-906.

76 Suzuki H, Kundig TM, Furlonger C, et al. Deregulated T cell activation and autoimmunity in mice lacking interleukin-2 receptor beta. Science 1995; 268:1472-1476.

77 Kramer S, Schimpl A, Hunig T. Immunopathology of interleukin (IL) 2-deficient mice: thymus dependence and suppression by thymus-dependent cells with an intact IL-2 gene. J Exp Med 1995; 182:1769-1776.

78 Horak I, Lohler J, Ma A, Smith KA. Interleukin-2 deficient mice: a new model to study autoimmunity and self-tolerance. Immunol Rev 1995; 148:35-44.

79 Malek TR, Yu A, Vincek V, Scibelli P, Kong L. CD4 regulatory T cells prevent lethal autoimmunity in IL-2Rbeta-deficient mice. Implications for the nonredundant function of IL-2. Immunity 2002; 17:167-178.

80 Klebb G, Autenrieth IB, Haber H, et al. Interleukin-2 is indispensable for development of immunological self-tolerance. Clin Immunol Immunopathol 1996; 81:282-286.

81 Almeida AR, Legrand N, Papiernik M, Freitas AA. Homeostasis of peripheral CD4+ T cells: IL-2R alpha and IL-2 shape a population of regulatory cells that controls CD4+ T cell numbers. J Immunol 2002; 169:4850-4860.

82 Setoguchi R, Hori S, Takahashi T, Sakaguchi S. Homeostatic maintenance of natural Foxp3(+) CD25(+) CD4(+) regulatory $\mathrm{T}$ cells by interleukin (IL)-2 and induction of autoimmune disease by IL-2 neutralization. J Exp Med 2005; 201:723-735.

83 Thornton AM, Donovan EE, Piccirillo CA, Shevach EM. Cutting edge: IL-2 is critically required for the in vitro activation of CD4+CD25+ T cell suppressor function. J Immunol 2004; 172:6519-6523.

84 Furtado GC, Curotto de Lafaille MA, Kutchukhidze N, Lafaille $\mathrm{JJ}$. Interleukin 2 signaling is required for CD4(+) regulatory $\mathrm{T}$ cell function. J Exp Med 2002; 196:851-857.

85 Curotto de Lafaille MA, Lino AC, Kutchukhidze N, Lafaille JJ. CD25- T cells generate CD25+Foxp3+ regulatory T cells by peripheral expansion. J Immunol 2004; 173:7259-7268.

86 Maloy KJ, Powrie F. Fueling regulation: IL-2 keeps CD4+ Treg cells fit. Nat Immunol 2005; 6:1071-1072.

87 Fontenot JD, Rasmussen JP, Gavin MA, Rudensky AY. A function for interleukin 2 in Foxp3-expressing regulatory T cells. Nat Immunol 2005; 6:1142-1151.

88 Yates J, Rovis F, Mitchell P, et al. The maintenance of human CD4+CD25+ regulatory T cell function: IL-2, IL-4, IL-7 and IL-15 preserve optimal suppressive potency in vitro. Int Immunol 2007; 19:785-799.

89 Schubert LA, Jeffery E, Zhang Y, Ramsdell F, Ziegler SF. Scurfin (FOXP3) acts as a repressor of transcription and regulates $\mathrm{T}$ cell activation. J Biol Chem 2001; 276:37672-37679.

$90 \mathrm{Wu}$ Y, Borde M, Heissmeyer V, et al. FOXP3 controls regulatory T cell function through cooperation with NFAT. Cell 2006; 126:375-387.

91 Rao A, Luo C, Hogan PG. Transcription factors of the NFAT family: regulation and function. Annu Rev Immunol 1997; 15:707-747.

92 Hogan PG, Chen L, Nardone J, Rao A. Transcriptional regulation by calcium, calcineurin, and NFAT. Genes Dev 2003; 17:22052232.
93 Crabtree GR, Olson EN. NFAT signaling: choreographing the social lives of cells. Cell 2002; 109(Suppl):S67-S79.

94 Macian F, Garcia-Cozar F, Im SH, et al. Transcriptional mechanisms underlying lymphocyte tolerance. Cell 2002; 109:719731.

95 Heissmeyer V, Macian F, Im SH, et al. Calcineurin imposes T cell unresponsiveness through targeted proteolysis of signaling proteins. Nat Immunol 2004; 5:255-265.

96 Wang B, Lin D, Li C, Tucker P. Multiple domains define the expression and regulatory properties of Foxp1 forkhead transcriptional repressors. J Biol Chem 2003; 278:24259-24268.

97 Bettelli E, Dastrange M, Oukka M. Foxp3 interacts with nuclear factor of activated T cells and NF-kappa B to repress cytokine gene expression and effector functions of T helper cells. Proc Natl Acad Sci USA 2005; 102:5138-5143.

98 Shevach EM. CD4+ CD25+ suppressor T cells: more questions than answers. Nat Rev Immunol 2002; 2:389-400.

99 von Boehmer H. Mechanisms of suppression by suppressor T cells. Nat Immunol 2005; 6:338-344.

100 Green EA, Gorelik L, McGregor CM, Tran EH, Flavell RA. CD4+CD25+ T regulatory cells control anti-islet CD8+ T cells through TGF-beta-TGF-beta receptor interactions in type 1 diabetes. Proc Natl Acad Sci USA 2003; 100:10878-10883.

101 Nakamura K, Kitani A, Strober W. Cell contact-dependent immunosuppression by $\mathrm{CD} 4(+) \mathrm{CD} 25(+)$ regulatory $\mathrm{T}$ cells is mediated by cell surface-bound transforming growth factor beta. J Exp Med 2001; 194:629-644.

102 Takahashi T, Tagami T, Yamazaki S, et al. Immunologic selftolerance maintained by $\mathrm{CD} 25(+) \mathrm{CD} 4(+)$ regulatory $\mathrm{T}$ cells constitutively expressing cytotoxic $\mathrm{T}$ lymphocyte-associated antigen 4. J Exp Med 2000; 192:303-310.

103 Paust S, Lu L, McCarty N, Cantor H. Engagement of B7 on effector $\mathrm{T}$ cells by regulatory $\mathrm{T}$ cells prevents autoimmune disease. Proc Natl Acad Sci USA 2004; 101:10398-10403.

104 Grossman WJ, Verbsky JW, Barchet W, et al. Human T regulatory cells can use the perforin pathway to cause autologous target cell death. Immunity 2004; 21:589-601.

105 Klein L, Khazaie K, von Boehmer H. In vivo dynamics of antigen-specific regulatory $\mathrm{T}$ cells not predicted from behavior in vitro. Proc Natl Acad Sci USA 2003; 100:8886-8891.

106Jordan MS, Riley MP, von Boehmer H, Caton AJ. Anergy and suppression regulate $\mathrm{CD} 4(+) \mathrm{T}$ cell responses to a self peptide. Eur J Immunol 2000; 30:136-144.

107 Asseman C, Mauze S, Leach MW, Coffman RL, Powrie F. An essential role for interleukin 10 in the function of regulatory T cells that inhibit intestinal inflammation. J Exp Med 1999; 190:995-1004.

108 Annacker O, Pimenta-Araujo R, Burlen-Defranoux O, et al. $\mathrm{CD} 25+\mathrm{CD} 4+\mathrm{T}$ cells regulate the expansion of peripheral CD4 T cells through the production of IL-10. J Immunol 2001; 166:3008-3018

109Suri-Payer E, Cantor H. Differential cytokine requirements for regulation of autoimmune gastritis and colitis by $\mathrm{CD} 4(+) \mathrm{CD} 25(+)$ T cells. J Autoimmun 2001; 16:115-123.

110 Van Parijs L, Abbas AK. Homeostasis and self-tolerance in the immune system: turning lymphocytes off. Science 1998; 280:243-248.

111 Malek TR, Bayer AL. Tolerance, not immunity, crucially depends on IL-2. Nat Rev Immunol 2004; 4:665-674. 
112 Barthlott T, Kassiotis G, Stockinger B. T cell regulation as a side effect of homeostasis and competition. J Exp Med 2003; 197:451-460.

113 von Boehmer H. Dynamics of suppressor T cells: in vivo veritas. J Exp Med 2003; 198:845-849.

114 Shevach EM. From vanilla to 28 flavors: multiple varieties of T regulatory cells. Immunity 2006; 25:195-201.

115 Fantini MC, Becker C, Monteleone G, et al. Cutting edge: TGF-beta induces a regulatory phenotype in CD4+CD25- T cells through Foxp3 induction and down-regulation of Smad7. J Immunol 2004; 172:5149-5153.

116 Chen W, Jin W, Hardegen N, et al. Conversion of peripheral
CD4+CD25- naive $\mathrm{T}$ cells to CD4+CD25+ regulatory $\mathrm{T}$ cells by TGF-beta induction of transcription factor Foxp3. J Exp Med 2003; 198:1875-1886.

117 Apostolou I, von Boehmer H. In vivo instruction of suppressor commitment in naive T cells. J Exp Med 2004; 199:14011408.

118 O'Garra A, Vieira P. Regulatory T cells and mechanisms of immune system control. Nat Med 2004; 10:801-805.

119 Carrier Y, Yuan J, Kuchroo VK, Weiner HL. Th3 cells in peripheral tolerance. I. Induction of Foxp3-positive regulatory T cells by Th3 cells derived from TGF-beta T cell-transgenic mice. J Immunol 2007; 178:179-185. 